\title{
Árboles de clasificación como herramienta para predecir dificultades financieras en empresas Latinoamericanas a través de sus razones contables
}

\author{
Classification trees as a tool to predict financial difficulties in Latin American \\ companies through its financial ratios
}

\author{
Norma Patricia Caro*, Mariana Guardiola y Pablo Ortiz \\ Universidad Nacional de Córdoba, Argentina
}

Recibido el 14 de febrero del 2016; aceptado el 2 de junio del 2017

Disponible en Internet el 11 de diciembre de 2017

\section{Resumen}

El análisis de la información contable de las empresas resulta relevante para la detección de situaciones de vulnerabilidad financiera. Si bien esta información suele estar disponible para aquellas empresas que cotizan en diferentes mercados bursátiles, generalmente no se cuenta con una caracterización explícita referida a su situación financiera (i.e. en crisis o sanas). En este contexto, el objetivo de este trabajo es analizar la incidencia de determinadas razones contables sobre la situación financiera de empresas de distintos mercados latinoamericanos: Chile, Perú y Argentina, en base a los estados contables correspondientes al período 2001-2011. A fin de detectar que las razones contables influyen primordialmente en las empresas en crisis, se aplicaron métodos recursivos: Árboles de Clasificación. De esta manera, se propicia contar con herramientas que permitan predecir el estado financiero de las empresas. En todos los mercados la rentabilidad resultó determinante con una fuerte incidencia en la clasificación de las empresas. Posteriormente, a efectos de analizar el impacto de otras razones, se la excluyó siendo las variables de mayor impacto disímiles entre los países. En el caso de Argentina, el tamaño de la empresa, el capital de trabajo y la rotación del activo resultaron las variables predictoras más importantes; en el caso de Chile fue el flujo de fondos operativos; y en el de Perú, la liquidez seguida por la rotación del activo.

Códigos JEL: G32, G33, C38

Palabras clave: árboles de clasificación, razones contables, crisis financiera, empresas latinoamericanas.

\footnotetext{
* Autor para correspondencia.

Correo electrónico: npatriciacaro@gmail.com (N.Patricia Caro)

La revisión por pares es responsabilidad de la Universidad Nacional Autónoma de México.
} 


\begin{abstract}
Analysis of accounting information of companies is relevant to the detection of situations of financial vulnerability. While this information is usually available for companies listed on various stock markets, generally not have an explicit characterization referred to its financial position (i.e. in crisis or healthy). In this context, the aim of this paper is to analyze the impact of same financial ratios on the financial situation of companies from different Latin American markets: Chile, Peru and Argentina, based on the financial statements in the period 2000 - 2011. In order to detect which financial ratios have primordial influence in companies in crisis, recursive methods were applied in this paper: Classification Trees. In this way, it is encouraged to have tools to predict the financial condition of the companies. In all markets, profitability was decisive with a strong impact on the classification of companies. Subsequently, in order to analyze the impact of other ratios, this variable was excluded, being more disparate the impact of other variables among countries. In the case of Argentina, the size of the company, working capital and asset turnover were the most important predictors; in the case of Chile was the operating cash flow; and that of Peru, liquidity followed by asset turnover.
\end{abstract}

JEL Classification: G32, G33, C38

Keywords: Classification trees, financial ratios, financial crisis, Latin American companies.

\title{
Introducción
}

La predicción de la insolvencia es uno de los temas centrales del análisis financiero, y ha suscitado el interés no sólo del ámbito académico, sino también de un amplio abanico de usuarios relacionados con el mundo empresarial. A partir de la década de 1960 (Beaver, 1966, 1968; Altman, 1968) se han aplicado diferentes metodologías, tanto univariadas como multivariadas (discriminante, logit, probit, etc.), las razones contables funcionan como variables explicativas, obteniendo resultados muy satisfactorios.

Altman (1993) utilizó árboles de clasificación (decisión) para la predicción de crisis empresarial en economías desarrolladas. En esta línea, el presente trabajo extiende este estudio a empresas que cotizan sus acciones en las bolsas de mercados latinoamericanos, mediante un modelo de predicción de insolvencia a fin de detectar qué las razones contables influyen primordialmente en las empresas en crisis. De esta manera se estudia la relación entre el estado de crisis de una empresa, definida como el estado de vulnerabilidad financiera producido por la obtención de pérdidas importantes que absorben el capital o el patrimonio neto, o bien por iniciar un proceso judicial de concurso preventivo o quiebra, y el comportamiento de ciertas razones contables. A partir de este trabajo, se propicia que empresas, entidades financieras, inversores, entre otros, puedan predecir el estado de vulnerabilidad financiera de las empresas según el comportamiento de algunos de los indicadores contables, al tiempo que constituye una motivación a continuar investigando en esta área de interés.

La determinación de puntos de corte a través de la aplicación de la técnica de árboles de decisión puede ser considerada como paso intermedio para la aplicación de modelos para datos longitudinales, cuyos primeros antecedentes en relación a su aplicación en países latinoamericanos, se encuentran en Caro, Díaz, et al. (2013) y Caro y Díaz (2015). De esta manera, estos puntos de corte permiten analizar los estados contables de las empresas y tomar decisiones en función del valor que asumen los mismos en cada ejercicio contable anual. 
Así, el presente trabajo contribuye a construir modelos de predicción que permitan una primera aproximación para dilucidar qué las razones financieras resultan significativos en la clasificación de las empresas, al tiempo que establece una regla que determina los umbrales a partir de los cuales una nueva empresa está en una situación de crisis o no.

Este trabajo ha sido estructurado de la siguiente forma: se comienza con una introducción en la que se establecen los objetivos y la motivación junto a una breve referencia de los primeros antecedentes en la literatura. A continuación se explica la muestra seleccionada y las variables medidas, siguiendo con la aplicación de técnicas de clasificación que nos permitirán evaluar si la información contable es relevante para la detección de situaciones de vulnerabilidad financiera. Finalmente, se exponen los resultados obtenidos, las conclusiones y consideraciones generales sobre el trabajo desarrollado.

\section{Antecedentes}

El problema de investigación que se aborda en este trabajo está contextualizado en la teoría de la utilidad de la información contable para la toma de decisiones. El cambio de enfoque que se produce a través de lo que se denominó la "revolución contable" (Beaver, 1981), hace cambiar la perspectiva de la medición de la ganancia económica a un enfoque “informativo". Entre los principales aspectos planteados por Beaver (1981), puede destacarse que la contabilidad ha sufrido cambios importantes desde la década de 1960, reflejándose en mayores requerimientos financieros de las empresas por parte de los organismos reguladores, lo que suscitó en un incremento en la cantidad de datos financieros que los usuarios necesitan, con el consiguiente cambio en los estados contables. El propósito de los estados financieros es, ahora, proveer información a los dueños del capital para poder predecir el beneficio (futuros flujos de fondos) y analizar la eficiencia y eficacia de la actuación gerencial.

La estrecha relación existente entre los indicadores económicos-financieros construidos a partir de los estados contables de las empresas y su situación futura, justifican su consideración en el estudio del pronóstico de riesgo de crisis financiera. Las empresas con dificultades financieras, aunque se las defina con diferentes términos tales como: empresas en crisis, insolventes, quebradas, fracasadas, en falencia, fallidas, entre otros, son aquellas que presentan signos de vulnerabilidad financiera y que se contraponen al concepto de empresas sanas o sin estas dificultades.

El problema de predicción del estado de crisis financiera, presente en las economías de todo el mundo, fue planteado inicialmente a comienzos de la década de 1930 donde las investigaciones sobre la situación financiera de las empresas se centraron en la tendencia de las razones. Esta etapa se caracterizó por la utilización de métodos descriptivos univariados, analizando el comportamiento de cada ratio en forma individual e indagando sobre las diferencias observadas entre grupos de empresas con problemas financieros y empresas sanas (Fitzpatrick, 1932; Winakor y Smith, 1935; Merwin, 1942).

A partir de la década de 1960 se desarrolló una etapa predictiva, donde se comenzó a analizar la significatividad estadística de cada índice entre los diferentes grupos de empresas, así como la capacidad que tienen para poder predecir la quiebra de una empresa. Beaver (1966, 1968) y Altman (1968) comenzaron con el desarrollo de modelos con estas características en economías desarrolladas, que se fueron adaptando y extendiendo a economías emergentes. Altman (1968) recurrió a la aplicación de herramientas estadísticas multivariadas, como el 
análisis discriminante, con el objetivo de clasificar empresas según su riesgo de quiebra; donde la metodología seguida se basó en un diseño apareado de muestras no aleatorias y la aplicación de métodos de discriminación lineal y cuadrática. Por su parte, en la década de 1980 aparecieron los primeros cuestionamientos al diseño no aleatorio (Ohlson, 1980; Zmijewski, 1984), acompañado de la modelación con regresión logística o modelo probit (Jones, 1987; Maddala, 1991; Ohlson, 1980).

Una de las críticas que se les puede atribuir a los modelos de Altman $(1968,1993)$ es que no resulta conveniente su aplicación en economías emergentes sin la correspondiente adecuación, ya que son modelos construidos con información de los estados contables de empresas en países desarrollados. Se ha probado que las tasas de error son altas (Sandin y Porporato, 2007 y que lo adecuado es la construcción de modelos con los datos propios de las empresas domésticas de cada país, considerando la información en el tiempo que se dispone de cada una de ellas.

Otra de las desventajas del uso de modelos de corte transversal (análisis discriminante, regresión logística, entre otros) es de tipo metodológica, es decir la verificación de ciertos supuestos para que los resultados obtenidos sean confiables.

En cuanto a la aplicación de técnicas de árboles de decisión, se destacan los trabajos de Altman (1993), Frydman, Altman, y Kao (1985) y Bonsón Ponte, Escobar Rodríguez, y Martín Zamora (1997). Los resultados indican que estas técnicas logran un mayor grado de acierto que las técnicas clásicas a la hora de clasificar a las empresas en solventes e insolventes, con mayor capacidad explicativa (Bonsón Ponte et al., 1997). Todos estos métodos trabajan con datos de corte transversal, es decir con un balance anual por cada empresa, pudiendo replicarlos uno, dos, tres, o más años antes de la manifestación de insolvencia.

Existen además otros métodos estadísticos aplicados a esta temática que trabajan con datos longitudinales, es decir datos correlacionados en el tiempo, tomando en consideración la información derivada de informes contables anuales de una misma empresa. En este sentido, Jones y Hensher $(2004,2007)$ aplicaron modelos mixtos, a empresas australianas, concluyendo que los indicadores explicativos de situaciones de crisis son aquellos que recogen información de efectivo o liquidez, flujo de fondos provenientes de las operaciones, capital de trabajo, rentabilidad, volumen del negocio, endeudamiento y capacidad de pago de los servicios de la deuda. Debido a que no se habían realizado investigaciones de esta índole en economías emergentes, Caro, Díaz, et al. (2013) utilizaron modelos mixtos para la predicción de crisis en empresas argentinas en la década de 1990 y, luego Caro y Díaz (2015) lo hicieron con empresas de la década del 2000.

Altman et al. (1977) fue uno de los pioneros en adaptar sus modelos de corte transversal para poder aplicarlos en economías emergentes. En Latinoamérica, surgieron así nuevos trabajos de investigación (Altman et al., 1979; Pascale, 1988; Swanson y Tybout, 1988; Romani Chocce et al., 2002; Sandin y Porporato, 2007; Zurita, 2008; Mongrut Montalván et al., 2011) con el objetivo de estudiar el comportamiento de los indicadores contables para predecir el riesgo de insolvencia.

Puede afirmarse que los modelos estadísticos responden de modo satisfactorio ante la necesidad de anticipar situaciones de vulnerabilidad financiera. Si bien en un comienzo se aplicaron modelos de clasificación supervisada para datos de corte transversal (considerando en el análisis un año, dos o tres antes de la manifestación de crisis, según las diferentes investigaciones), en la actualidad se consideran más apropiados, a los efectos de explicar y predecir la crisis financiera, a aquellos que contemplan la historia de las empresas. 
En los últimos años se observa un avance importante en la aplicación de metodologías en múltiples áreas de las ciencias económicas. De Llano Monelos, et al. (2014) han desarrollado modelos para diagnosticar y pronosticar el fracaso empresarial con base en variables financieras aplicando tres metodologías de análisis: discriminante, logit y lineal multivariante. También utilizaron programación matemática $\mathrm{DEA}^{1}$ (Análisis envolvente de Datos) para fundamentar la determinación del fracaso, metodología que aportó puntos significativos en cuanto a su aplicabilidad al pronóstico del fallo empresarial. No obstante, estas técnicas presentan el inconveniente de que parten de hipótesis más o menos restrictivas acerca de las propiedades distribucionales de las variables explicativas que, especialmente en el caso de la información contable, no se suelen cumplir. En un intento de superar esta limitación surge el empleo de técnicas procedentes del campo de la Inteligencia Artificial ya que, debido a su carácter no paramétrico, no precisan de hipótesis preestablecidas sobre las variables de partida. Dentro de estas técnicas, las más utilizadas en el análisis de la solvencia empresarial son los sistemas de redes neuronales, los conjuntos aproximados (Rough Sets en terminología anglosajona), los algoritmos genéticos o los sistemas de inducción de reglas y árboles de decisión.

\section{Muestra y datos}

Este trabajo contribuye en términos generales a fomentar la investigación en el área de la contabilidad y administración, y en particular, dirigida a los modelos de predicción de crisis a través del uso de modelos estadísticos avanzados, que permiten no sólo detectar situaciones futuras de vulnerabilidad financiera sino que también propicia la adopción de cursos de acción adecuados para prevenir dichas situaciones en el escenario de países latinoamericanos.

El principal objetivo de los modelos de predicción, en economías emergentes en particular, es la detección oportuna de empresas que puedan fracasar en el futuro y su utilidad práctica solo es de interés cuando dichos modelos son capaces de distinguir entre empresas que no fracasan y empresas que si fracasan.

La población objeto de análisis está conformada por las empresas argentinas, chilenas y peruanas que cotizaron sus acciones en el período 2001-2011 en las respectivas Bolsas, a saber:

a) La Bolsa de Comercio de Buenos Aires (BCBA), fundada el 10 de julio de 1854, es la más importante de Argentina, constituyéndose como el centro financiero del país. Sus principales transacciones se centran en acciones de empresas, bonos, divisas y futuros. Se trata de una asociación civil sin fines de lucro, dirigida por representantes de los diferentes sectores del empresariado, regulada por la Ley $\mathrm{N}^{\circ} 17.811$ y supervisada por la Comisión Nacional de Valores. La particularidad del sistema bursátil argentino es que la Bolsa y el Mercado de Valores son dos instituciones distintas, cada una con funciones y roles específicos, a diferencia de la mayoría de los sistemas bursátiles del resto del mundo, que tienen una única institución denominada Bolsa de Valores. Las empresas que desean cotizar sus acciones en la Bolsa de Comercio de Buenos Aires deben informar trimestralmente sus estados contables y aportar toda información relevante.

b) La Bolsa de Comercio de Santiago (BCS), fundada el 27 de noviembre de 1893, es el principal centro de operaciones bursátiles de Chile. Sus transacciones son acciones, bonos y divisas extranjeras. La Bolsa es miembro fundador de la Federación Iberoamericana de Bolsas de Valores (FIAB) desde 1973.

\footnotetext{
${ }^{1}$ DEA: Data Envelopment Analysis.
} 
Como indicadores, puede mencionarse el Índice General de Precios de Acciones (IGPA), creado en 1958 y compuesto de la mayoría de las acciones y de revisión anual, y el Índice de Precio Selectivo de Acciones (IPSA) que corresponde a las 40 acciones más transadas.

c) La Bolsa de Valores de Lima (BVL) resurgió bajo el nombre de Bolsa Comercial de Lima en 1898. El mercado bursátil está regulado por la Ley de Mercado de Valores, Decreto Legislativo $\mathrm{N}^{\circ} 861$ y el ente regulador es la Superintendencia del Mercado de Valores (SMV), organismo técnico especializado, que tiene por finalidad velar por la protección de los inversionistas, la eficiencia y transparencia de los mercados bajo su supervisión, la correcta formación de precios y la difusión de toda la información necesaria para tales propósitos. La Bolsa de Valores de Lima (BVL) es la única entidad autorizada para funcionar en el país y tiene por objeto facilitar las negociaciones de valores inscriptos.

Los motivos por los cuales no fueron considerados otros países latinoamericanos se debe a que, algunos de ellos no poseen Bolsa (Uruguay), o no proporcionan el dato de la vulnerabilidad financiera (Colombia y Brasil).

El horizonte temporal de esta investigación comprende el período 2001 - 2011, en función de la disponibilidad de datos que no se encuentren distorsionados por situaciones de inestabilidad económica. Con posterioridad a ese período, estos países comenzaron a implementar las Nuevas Normas Internacionales de Contabilidad que establecen algunos criterios diferentes en cuanto a valuación y exposición de la información. Por esto no es conveniente utilizar informes contables de períodos posteriores a la implementación de dichas normas ya que sus resultados no resultarían comparables.

Dentro del total de empresas en cada bolsa, se identificaron como empresas en crisis a aquellas que han cotizado en rueda reducida en el período de análisis; o bien se haya informado, a través de noticias de la Bolsa, la suspensión de la cotización de sus acciones por haber entrado en cesación de pagos o haberse declarado su quiebra; o bien, por la cantidad de pérdidas obtenidas que absorben parte de su capital o patrimonio neto. La muestra (Tabla 1) se encuentra constituida por los estados contables de las empresas en crisis junto con los de una selección de empresas sanas, entendidas como aquellas firmas que no manifiestan estos signos de vulnerabilidad, disponibles para cada uno de los mercados en el período considerado. Particularmente, para las empresas en crisis se tomó el estado contable anual anterior al año en que se detectó su vulnerabilidad financiera y para las sanas, el del último período disponible. Se excluyeron bancos, compañías financieras y de seguros, ya que poseen una regulación específica y su análisis no sería comparable con las demás (Altman, 1968).

A raíz de que la cantidad de empresas que cotizan en Bolsa en los mercados latinoamericanos no es numerosa, no se pudo realizar un análisis por sector, ya que algunos quedaron no representados y otros tenían muy pocas empresas, por lo que se dividió a las mismas por tipo de actividad (industrial, comercial y de servicios), lo que no resultó estadísticamente significativo, por lo que no fue considerado en esta investigación.

Tabla 1

Cantidad de empresas por mercado

\begin{tabular}{lccc}
\hline Estado & Chile & Perú & Argentina \\
\hline Sana & 158 & 37 & 44 \\
En crisis & 33 & 13 & 13 \\
Total & 191 & 50 & 57 \\
\hline
\end{tabular}

Fuente: Elaboración propia 
La variable dependiente es el estado de la empresa, con dificultades financieras (crisis) y sin ellas (sanas). Por su parte, las variables predictoras se encuentran definidas por razones contables, los cuales son indicadores construidos a partir de la información de los estados contables presentados por las empresas, que han sido empleados en estudios previos. Estudios más recientes han incluido razones no contempladas con anterioridad, como el índice de flujo de fondos operativos, por lo que es relevante para este trabajo incluirlo.

Los indicadores considerados en este trabajo son los que utiliza, Caro, Díaz, et al. (2013) basados en Altman (1968) y Jones y Hensher (2004), siendo las razones más utilizadas y que han sido validadas en múltiples aplicaciones en diferentes mercados:

- Ratio de Flujo de Fondos (FF_AT): mide la posición de efectivo originado por las actividades operativas respecto al total del activo.

- Ratio de Liquidez (E_AT): mide la proporción de los recursos más líquidos de la empresa (disponibilidades e inversiones que pueden hacerse efectivas dentro de las 24 horas) sobre el total de activo.

- Ratio de Rotación del Activo Total (V_AT): es el coeficiente que mide la relación entre las ventas y el activo total.

- Ratio de Endeudamiento (D_PN): mide la relación de las deudas (capital de terceros) sobre el capital aportado por los dueños de la empresa.

- Ratio de Capital de trabajo (CT_AT): expresa el grado de fluidez de los activos e indica en qué medida la inmovilización del activo total resulta neutralizada por el capital de trabajo.

- Ratio de Rentabilidad económica (GE_AT): mide la rentabilidad en función de las ganancias de la explotación.

- Tamaño de la empresa (LNA): medido como el logaritmo natural del activo total.

\section{Método}

Altman (1993) fue uno de los iniciadores en el uso de metodologías clásicas (como el análisis discriminante) y no clásicas (como las redes neuronales y los árboles de clasificación y/o decisión) en economías desarrolladas.

En este trabajo se aplica una metodología basada en el algoritmo de inducción de reglas denominado CHAID (Chi-squared Automatic Interaction Detector), que fueron desarrollados por (Kass, 1980; Breiman et al., 1984)Esta técnica estadística de segmentación genera un árbol de reglas que especifica los distintos segmentos en que se divide la muestra en relación a la variable dependiente, permitiendo clasificar a las empresas de acuerdo a los diferentes valores que presentan sus razones contables.

Aunque son muchos los algoritmos capaces de generar reglas basados en árboles de clasificación, la elección del CHAID se basa tanto por su amplia difusión como por sus importantes beneficios.

En primer lugar, no está basado en ninguna distribución de probabilidad, empleando solamente los test de bondad de la Chi-cuadrado (derivados de tablas de contingencia) que, con un tamaño muestral aceptable, casi siempre produce buenos resultados. En segundo lugar, también permite determinar una variable a maximizar, lo que es deseable y no siempre posible con otras técnicas de segmentación. En tercer lugar, la clasificación mediante segmentos es relativamente fácil de analizar, ya que aporta reglas intuitivas sencillas de interpretar por usuarios no expertos, algo que no siempre ocurre cuando se trabaja con otras metodologías. 
En cuarto lugar, la técnica asegura que los segmentos siempre tienen significado estadístico, es decir, que son todos diferentes y los mejores posibles en base a los datos muestrales. Consecuentemente, las clasificaciones realizadas con las reglas encontradas son mutuamente excluyentes, y por tanto el árbol de decisión proporciona una única respuesta basada en el cálculo de las probabilidades de pertenecer a una cierta clase. Finalmente, CHAID es un algoritmo no binario, es decir, capaz de construir más de dos divisiones de los datos de acuerdo a las categorías a explicar en cada nodo.

El algoritmo CHAID consta de varios pasos en su aplicación hasta la obtención de las reglas (Breiman et al., 1984):

1. División de las variables explicativas continuas: Estas variables son discretizadas y divididas en un conjunto de categorías.

2. Fusión de categorías de las variables explicativas: Este algoritmo fusiona aquellos valores de las variables explicativas que son estadísticamente homogéneos respecto a la variable dependiente, y mantiene separados aquellos otros que son heterogéneos. Cada categoría final obtenida de una variable explicativa $X$ representa un nodo hijo si luego la variable es utilizada para dividir el árbol. Para cada variable explicativa X, el algoritmo encuentra la pareja de categorías de $\mathrm{X}$ que tienen menos diferencias significativas con respecto a la variable dependiente $\mathrm{Y}$, es decir, con mayor $p$-value, el cual se obtiene a partir del test de la Chi-cuadrado

3. División de nodos: cada variable es evaluada en su asociación con la variable dependiente, según el $p$-value obtenido con el test estadístico, de modo que el algoritmo selecciona el mejor predictor para formar la primera división del árbol, es decir, la variable explicativa con mayor asociación con la variable dependiente (aquella cuyo test Chi-cuadrado proporcionó el menor $p$-value). Si este valor es menor o igual que el umbral de división fijado por el usuario, entonces la variable se utiliza como variable de división para el nodo en cuestión, y cada una de las categorías fusionadas de la variable de división define un nodo hijo. Una vez dividido el nodo en cuestión, los nodos hijos son examinados para ver si permiten más divisiones con la aplicación de este proceso de fusión/división. Este proceso continua recursivamente hasta que el árbol queda configurado y no se pueden realizar más divisiones. El nivel de confianza de cada regla (nodo terminal) representa la proporción de registros de cada regla que pertenecen a la categoría $j$ seleccionada, mientras que de manera similar, el nivel de confianza de un conjunto de reglas puede definirse como la proporción de casos de cada regla que pertenecen a la categoría $j$ dada.

4. Índice: el índice de cada una de las reglas obtenidas para una categoría $j$ se obtiene como el ratio entre el nivel de confianza de cada regla o nodo terminal y el nivel de confianza de la categoría $j$ en la muestra total. La ganancia para cada nodo terminal $(t)$ se define en términos absolutos como el número de casos en la categoría $j$ seleccionada. Para un conjunto de reglas o nodos terminales, y en términos relativos, la ganancia representa el porcentaje de casos en la categoría $j$, y se puede notar donde es igual a 1 si el caso posee la categoría $j$, y 0 en caso contrario. 


\section{Resultados $^{2}$}

En los tres mercados la rentabilidad resultó determinante con una fuerte incidencia en la clasificación de las empresas. Posteriormente, a efectos de analizar el impacto de otras razones que se veían soslayadas, se le excluyó, obteniendo resultados relativamente más disimiles entre los países.

En el caso de Argentina, donde se obtuvo el árbol de mayor complejidad en cuanto a la cantidad de ramificaciones (Figura 1), el tamaño fue la principal variable predictora para el estado de las empresas. El 94,1\% de empresas de mayor tamaño, con un ratio LNA superior a 19.68\%, son sanas. Adicionalmente, de este grupo de empresas, el $100 \%$ con un ratio de endeudamiento (D_PN) inferior a $198.31 \%$ son sanas.

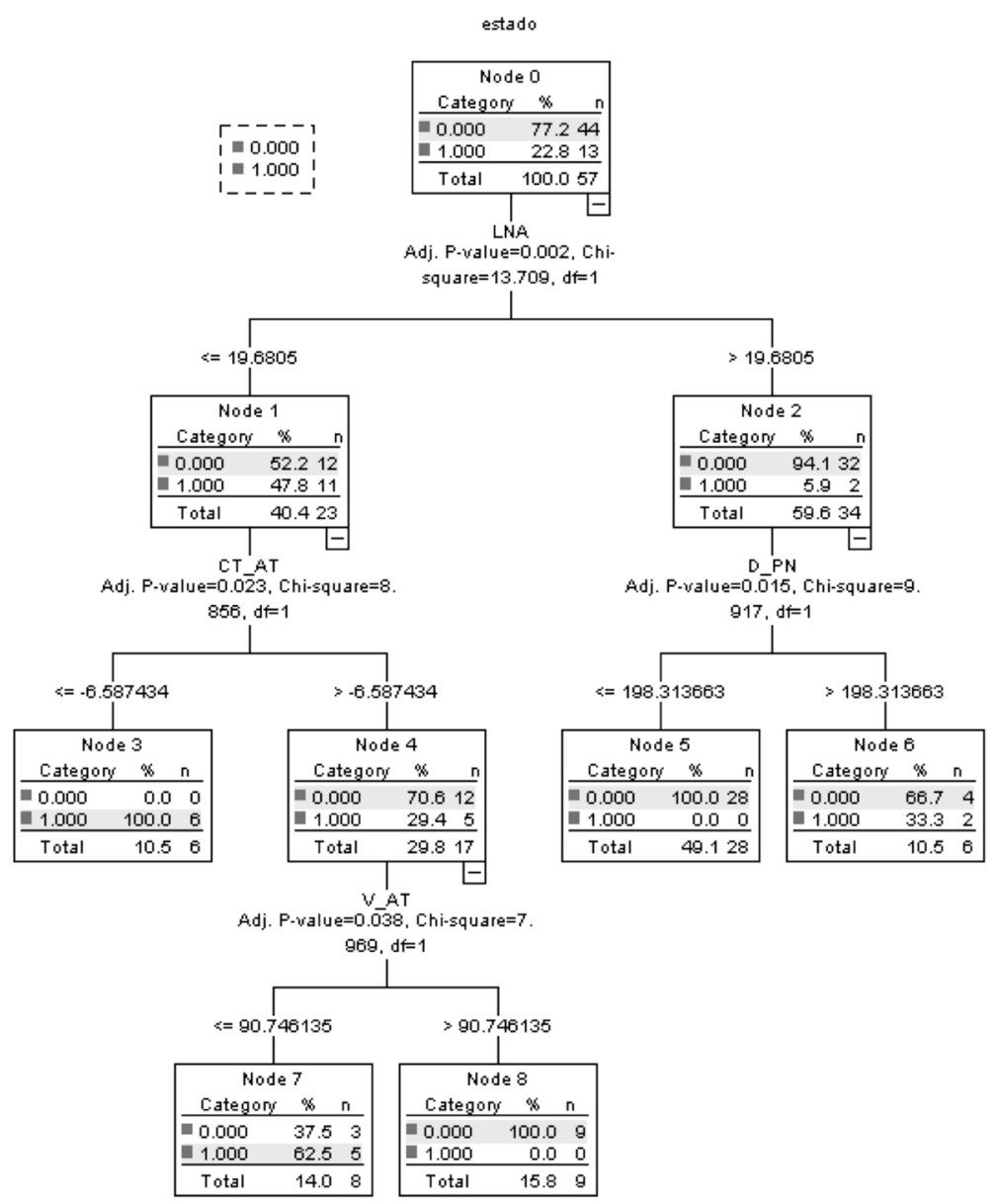

Figura 1. Árbol de Clasificación - Argentina Fuente: Elaboración propia

\footnotetext{
${ }^{2}$ Los datos fueron procesados con SPSS (Statistical Package for the Social Sciences).
} 
Por otra parte, las empresas de menor tamaño se encuentran distribuidas de manera bastante equitativa entre las categorías, siendo $47.8 \%$ en crisis; aunque de éstas, el $100 \%$ que tienen un ratio de capital de trabajo (CT_AT) inferior a $-6.59 \%$ se corresponden a esa categoría. En cuanto a la totalidad de empresas que devienen con estas características tienen una rotación de activos (V_AT) menor a $90.75 \%$.

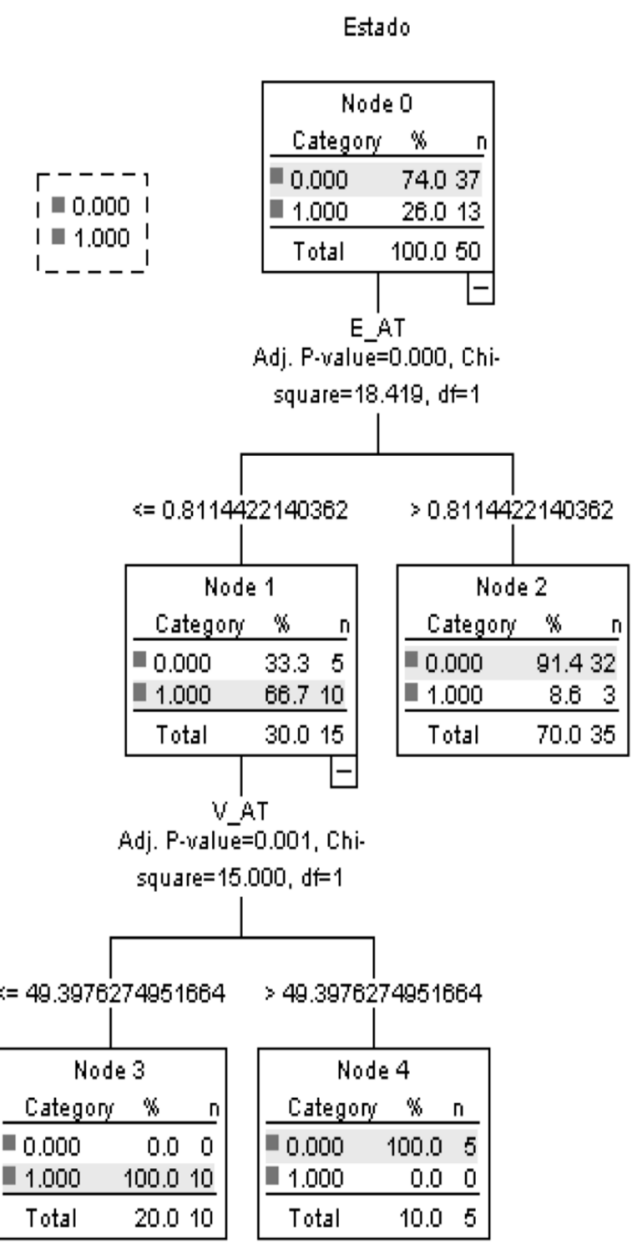

Figura 2. Árbol de Clasificación - Perú

Fuente: Elaboración propia

En cuanto a Perú, la liquidez resultó la variable predictora más relevante (Figura 2): el $91.4 \%$ de empresas con un ratio superior a $0.81 \%$ son sanas, mientras que el $66.7 \%$ con un ratio inferior a ese valor son empresas en crisis. Adicionalmente, este grupo de empresa de menor liquidez queda totalmente determinado por la rotación de activos, puesto que, la totalidad de empresas con este ratio inferior a $49.4 \%$ están crisis, mientras que las que superan esa magnitud son sanas. 


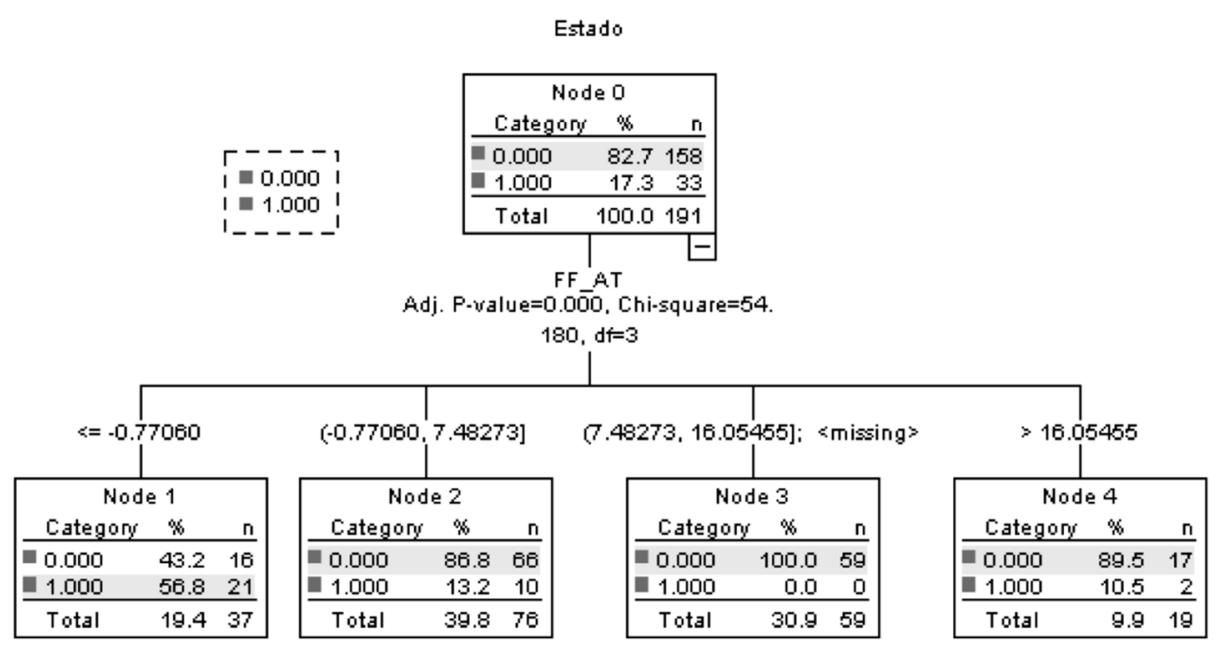

Figura 3. Árbol de Clasificación - Chile Fuente: Elaboración propia

Con respecto a Chile, el flujo de fondos resultó la variable predictora dominante (Figura 3). De la totalidad de empresas en crisis de la muestra de este país, el 64\% tienen un nivel de flujo de fondos (FF_AT) inferior a $-0.77 \%$ (constituyendo el $56.8 \%$ de las empresas con esta característica), mientras que el $92 \%$ de empresas con un ratio superior a esa magnitud, son empresas sanas.

En relación a la bondad del funcionamiento del modelo en los diferentes países, considerando el porcentaje de clasificación correcta (Tabla 2), Perú fue el de mejor performance tanto a nivel general (94\%) como en la clasificación específica de las empresas sanas (100\%), alcanzando además $76.9 \%$ en el caso de empresas en crisis. Seguidamente, las empresas de Argentina fueron clasificadas correctamente en un $91.2 \%$, junto al mejor desempeño en cuanto a las empresas en crisis, con un $84.6 \%$ de clasificación correcta. Finalmente, la tasa de clasificación global de las empresas chilenas fue muy buena, alcanzando un $85.3 \%$, aunque en relación a las empresas en crisis la performance resultó un poco inferior, $63.6 \%$.

Tabla 2

Porcentajes de clasificación correcta por mercado

\begin{tabular}{llll}
\hline Estado & Argentina & Chile & Perú \\
\hline Empresas sanas (0) & $93.2 \%$ & $89.9 \%$ & $100 \%$ \\
Empresas en crisis (1) & $84.6 \%$ & $63.6 \%$ & $76.9 \%$ \\
Porcentaje Global & $91.2 \%$ & $85.3 \%$ & $94 \%$ \\
\hline
\end{tabular}

Fuente: Elaboración propia 
Tabla 3

Intervalos para predecir un estado de crisis financiera por mercado

\begin{tabular}{lcccc}
\hline Ratios & Chile & Perú & \multicolumn{2}{c}{ Argentina } \\
\hline Tamaño & --- & --- & $\leq 19.68$ & --- \\
Capital de trabajo & --- & --- & $\leq-6.59$ & $>-6.59$ \\
Liquidez & --- & $\leq 0.81$ & --- & --- \\
Rotación de Activos & --- & $\leq 49.4$ & --- & $\leq 90.74$ \\
Flujo de Fondos & $\leq-0.77$ & --- & --- \\
\hline
\end{tabular}

Fuente: Elaboración propia

Las reglas obtenidas identifican perfiles de alta o baja performance, lo que indicaría que si una determinada empresa posee cierto comportamiento en sus razones, podría estar en peligro de crisis financiera. En función de los árboles de clasificación obtenidos (Figuras 1 a 3) se calcularon los intervalos de las diferentes razones a partir de las cuales empresarios, bancos o inversores, pueden determinar en qué situación financiera se encuentra la empresa de interés (Tabla 3).

De la lectura de la tabla precedente se desprende que una empresa chilena tiene una alta posibilidad de presentar problemas financieros si su ratio referido al flujo de fondos asume un valor menor o igual a - 0.77 . Mientras que en el caso de las empresas peruanas, la probabilidad de atravesar procesos de crisis es mayor si su ratio de liquidez no supera el valor 0.81 y la rotación del activo es a lo sumo 49.4. Respecto a las empresas argentinas con mayores probabilidades de experimentar procesos de vulnerabilidad financiera, se pueden identificar dos tipos: empresas cuyas razones de tamaño y capital de trabajo arrojen valores menores o iguales a 19.68 y -6.59, respectivamente y empresas con un índice de capital de trabajo superior a dicha magnitud pero con rotación del activo no mayor a 90.74 .

\section{Conclusiones}

En esta investigación se han presentado las principales características económico-financieras de las empresas cotizantes en las Bolsas latinoamericanas que han formado parte de la misma. Las reglas o perfiles obtenidos nos han permitido caracterizar a las empresas de acuerdo a las razones de sus estados contables. Sin embargo, el principal valor agregado de este trabajo no ha sido confirmar empíricamente las variables principales a tener en cuenta en la planificación financiera, sino determinar los niveles o intervalos cuantitativos a marcar como objetivo en cada una de ellas, propiciando la previsión de inconvenientes financieros.

Se concluye así que la aplicación de estos métodos estadísticos permite identificar empresas con problemas financieros, lo cual cobra relevancia en la modelación y predicción de este tipo de riesgo.

De este modo, en el presente trabajo se ha podido recomendar una regla que permite, con adecuados valores en las razones, predecir un estado de vulnerabilidad para que cada empresa, atendiendo a sus circunstancias particulares (internas y externas), pueda realizar su planificación financiera de acuerdo a las reglas que considere alcanzables y sostenibles. 


\section{Referencias}

Altman, E. I. (1968). Financial ratios, discriminant analysis and the prediction of corporate bankruptcy. The Journal of Finance, 23(4), 589-609. https://doi.org/10.2307/2978933

Altman, E. I. (1993). Corporate Financial Distress and Bankruptcy (J. W. a. Sons Ed. Second ed.). New York.

Altman, E. I., Baidya, T. K. \& Dias, L. M. R. (1979). Assessing potential financial problems for firms in Brazil. Journal of International Business Studies, 10(2), 9-24. https://doi.org/10.1057/palgrave.jibs.8490787

Altman, E. I., Haldeman, R. G. \& Narayanan, P. (1977). ZETATM analysis A new model to identify bankruptcy risk of corporations. Journal of Banking \& Finance, 1(1), 29-54. https://doi.org/10.1016/0378-4266(77)90017-6

Beaver, W. H. (1966). Financial ratios as predictors of failure. Journal of Accounting Research, 71-111. https://doi. $\operatorname{org} / 10.2307 / 2490171$

Beaver, W. H. (1968). Alternative accounting measures as predictors of failure. The Accounting Review, 43(1), 113-122.

Beaver, W. (1981) Financial reporting: an Accounting Revolution Prentice Hall.

Bonsón Ponte, E., Escobar Rodríguez, T. \& Martín Zamora, M. (1997). Decision tree induction systems. Applications in Accounting and Finance. Paper presented at the Intelligent Technologies in Accounting and Business. Proceedings of the III International Meeting on Artificial Intelligence in Accounting, Finance and Tax. Huelva.

Breiman, L., Friedman, J., Olshen, R. \& Stone, C. (1984). Classification and Regression Trees (Monterey, CA: Wadsworth and Brooks/Cole).

Caro, N. P. \& Díaz, M. (2015). Factores determinantes para predecir la crisis financiera en empresas argentinas/Determinants factors in predicting the financial crisis in argentine companies. Cuadernos de Estudios Empresariales, 25 , 29. https://doi.org/10.5209/rev_cese.2015.v25.53631

Caro, N. P., Díaz, M. \& Porporato, M. (2013). Predicción de quiebras empresariales en economías emergentes: uso de un modelo logístico mixto. Revista de Métodos Cuantitativos para la Economía y la Empresa, 16, $200-215$. Disponible en http://www.redalyc.org/html/2331/233129568008/ Consultado: 26/10/2017

De Llano Monelos, P., Sánchez, C. P. \& López, M. R. (2014). DEA as a business failure prediction tool Application to the case of galician SMEs. Contaduría y Administración, 59(2), 65-96. https://doi.org/10.1016/s0186-1042(14)712550

Fitzpatrick, P. (1932). A Comparison of ratios of successful industrial enterprises with those of failed firms. Certified Public Accountant, 1932. October, November, and December, 598-605.

Frydman, H., Altman, E. I. \& Kao, D. L. (1985). Introducing recursive partitioning for financial classification: the case of financial distress. The Journal of Finance, 40(1), 269-291. https://doi.org/10.2307/2328060

Jones, F. L. (1987). Current techniques in bankruptcy prediction. Journal of Accounting Literature, 6(1), $131-164$.

Jones, S. \& Hensher, D. A. (2004). Predicting firm financial distress: A mixed logit model. The Accounting Review, 79(4), 1011-1038. https://doi.org/10.2308/accr.2004.79.4.1011

Jones, S. \& Hensher, D. A. (2007). Modelling corporate failure: A multinomial nested logit analysis for unordered outcomes. The British Accounting Review, 39(1), 89-107. https://doi.org/10.1016/j.bar.2006.12.003

Kass, G. V. (1980). An exploratory technique for investigating large quantities of categorical data. Applied Statistics, 119-127. https://doi.org/10.2307/2986296

Maddala, G. S. (1991). A perspective on the use of limited-dependent and qualitative variables models in accounting research. The Accounting Review, 66(4), 788-807.

Merwin, C. L. (1942). Financing Small Corporations in Five Manufacturing Industries, 1926-36. Retrieved from

Mongrut Montalván, S. M., O’Shee, D. F., Delgado, F. A. \& Yamashiro, M. A. (2011). Determinantes de la insolvencia empresarial en el Perú. Academia(47), 126. Disponible en http://www.redalyc.org/html/716/71618917009/ Consultado: 26/10/2017

Ohlson, J. A. (1980). Financial ratios and the probabilistic prediction of bankruptcy. Journal of Accounting Research, 109-131. https://doi.org/10.2307/2490395

Pascale, R. (1988). A multivariate model to predict firm financial problems: the case of Uruguay. Studies in Banking and Finance, 7, 171-182. 
Romani Chocce, G. A., Aroca González, P., Aguirre Aguirre, N., Leiton Vega, P. \& Muñoz Carrazana, J. (2002). Modelos de clasificación y predicción de quiebra de empresas: Una aplicación a empresas chilenas. Paper presented at the Forum Empresarial. Disponible en http://www.redalyc.org/html/631/63170102/ Consultado: 26/10/2017

Sandin, A. R. \& Porporato, M. (2008). Corporate bankruptcy prediction models applied to emerging economies: Evidence from Argentina in the years 1991-1998. International Journal of Commerce and Management, 17(4), 295311. https://doi.org/10.1108/10569210710844372

Swanson, E. \& Tybout, J. (1988). Industrial bankruptcy determinants in Argentina. Studies in Banking and Finance, 7, 1-25.

Winakor, A. \& Smith, R. (1935). Changes in financial structure of unsuccessful industrial companies. Bureau of Business Research. Bulletin, 51.

Zmijewski, M. E. (1984). Methodological issues related to the estimation of financial distress prediction models. Journal of Accounting Research, 59-82. https://doi.org/10.2307/2490859

Zurita, F. (2008). La predicción de la insolvencia de empresas chilenas. Economía Chilena, 11(1), 93-116. 